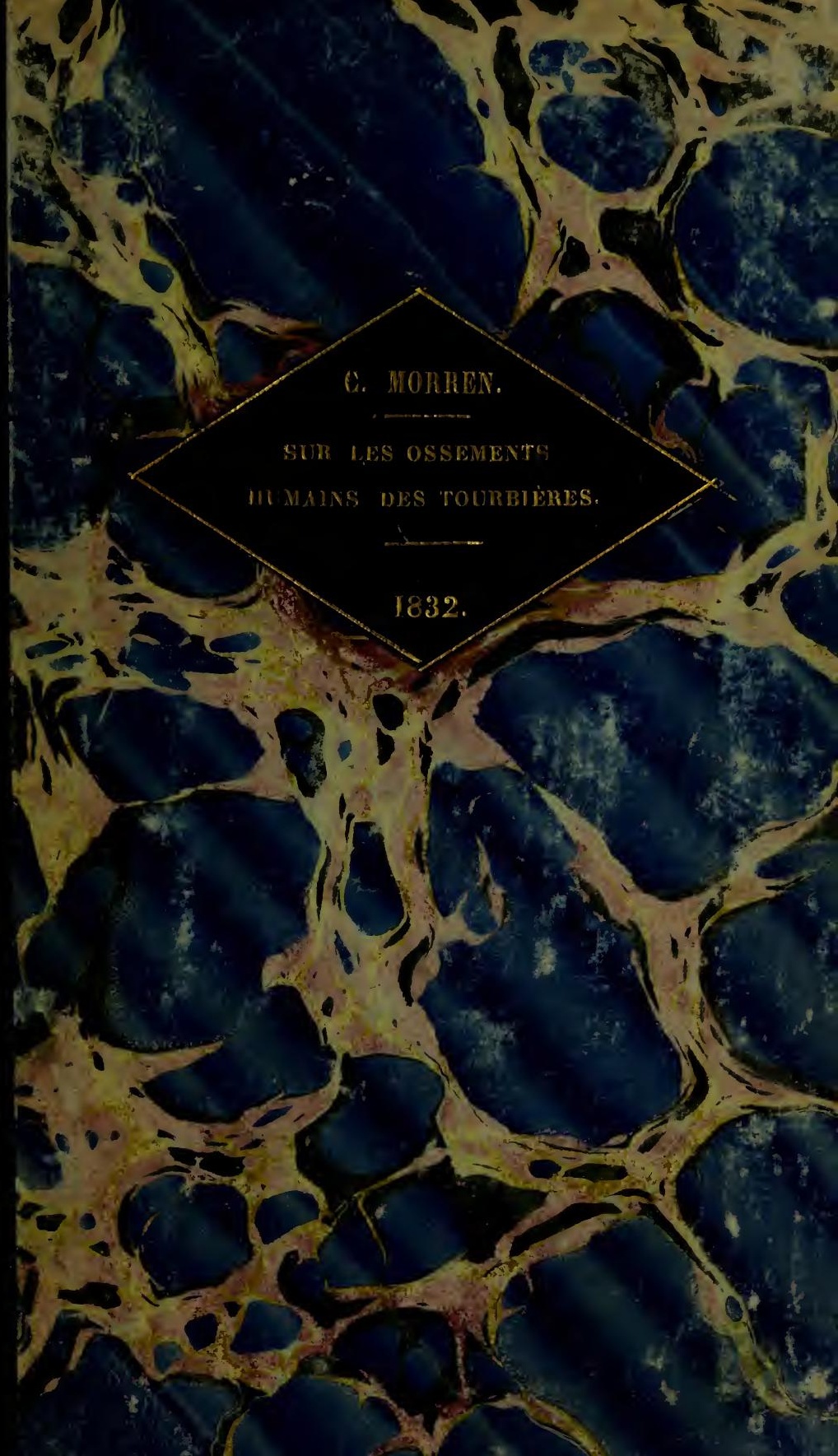




\section{$M a-M$}

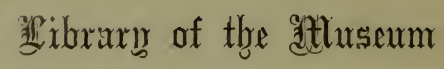

OF

\section{COMPARATIVE ZOÖLOGY,}

AT HARVARd COLLEGE, CANBBRIDGe, ILASS.

- Founder by pribate subscription, in 1861.

DR. L. DE KONINCK'S LIBRARY.

No. $164 \%$. 



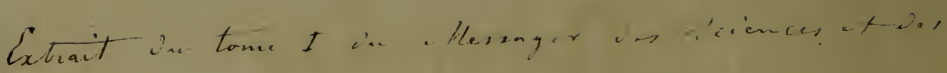

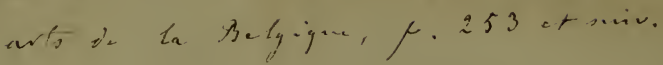




\section{Altémoire}

\section{SUR LES \\ OSSEMENS HUMAYNS DES TOURBIIRES}

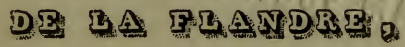

PAR

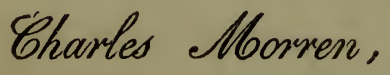

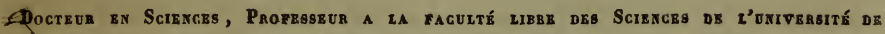

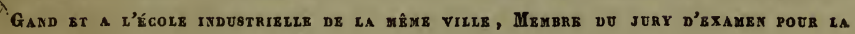

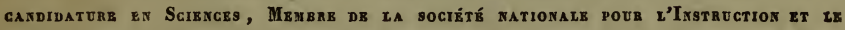
Bonheur du peuple, de ta sociétí Gíologiquz de France, de celle des Scisncis Physques, Chimiquas ex Arts Industriels dr Paris, dz ta socí́tí hoyale de Botanique et d'Agriculturb de Gand, de celle des Arts at Seirgces d'Utrecht,

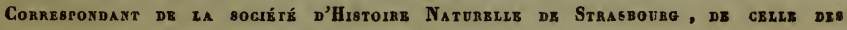

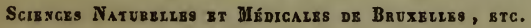

Mystérieux abîme où l'esprit so confond! A quelques pieds sous terre un silence profond, Et tant de bruit à la surface!

Victon Hugo. Feuilles d'automne. IV.

\section{$\mathfrak{G} \mathfrak{n} \mathfrak{d}$}

IMPRIMERIE DE D, J. VANDERHAEGHEN, IMPRIMECR DE L'UNIVERSITE, ETC, RuE BABSB N.० 21.

1832. 



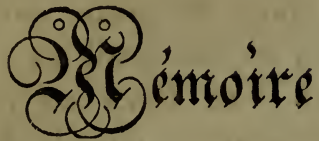

\section{SUR LES OSSEMENS HUMAINS DES TOURBIÈRE}

\section{DE LA FLANDRE.}

Les historiens n'ont pas attendu la naissance de la géologie pour s'occuper des hommes fossiles ou de ce qu'ils appelaient des hommes d'avant le déluge, et les idées superstitieuses, se mêlant à tout ce qui sort de l'ordre habituel des choses, il n'est pas d'absurdités qu'on n'ait débitées sur le compte de ces prétendus contemporains d'Adam. Pour les juifs, ce père du genre humain n'était rien moins qu'un géant, et ses enfans, quoique déjà plus petits que lui, étaient pourtant, selon eux, d'une taille beaucoup plus élevée que la nôtre. Le paganisme et les textes sacrés se ressemblent sur ce point que tous deux attribuent aux premiers hommes une grandeur extraordinaire. Il n'en fallut pas davantage pour attribuer à l'espèce humaine tous les grands ossemens que les extractions, les éboulemens ou le ravage des eaux faisaient découvrir dans la terre. Les payens y voyaient des os de dieux ou de héros; en Crète on avait déterré les os d'Orion, à Tégée ceux d'Oreste, à l'île de Lade ceux d'Ajax, à Trapani ceux de Poly phème. Des chrétiens trop crédules les prenaient pour des os de saints; à l'église de Valence, on montrait comme une relique une dent de S.t Christophe : cette dent était celle d'un éléphant, et les os des héros de la mythologie avaient appartenu comme elle à des individus de cette grande espèce de pachyderme. Nos ancêtres avaient des idées pareilles et croyaient à 


\section{(2)}

l'existence d'une race de géans qui avait vécu aux environs d'Anvers, où l'on avait découvert, disait-on, de leurs ossemens. Van Gorp ou Goropius Becanus détruisit déjà cette opinion dans le XVI.me siècle ; ces os étaient encore d'éléphans. Plus tard, ce fut non seulement la race éléphantique qui eut l'honneur de voir prendre ses restes pour ceux de l'orgueilleuse espèce humaine, mais encore celle des boeufs, des ânes, des tortues et jusqu'à celle de grands crapauds à queue. Étrange effet de l'ignorance! Pour elle, c'était ennoblir le genre humain que de l'avilir jusque là. Un anglais, M.r Bowles, croyait avoir vu à Concud des jambes et des cuisses d'hommes et de femmes, c'étaient des jambes d'ânes et de boeufs. Spallanzani prenait les ossemens de l'île de Cérigo pour des restes d'hommes, c'en étaient de quadrupèdes. A Aix, en Provence, on avait trouvé des têtes d'hommes, c'étaient des caparaces de tortues. Scheuchzer croyait décrire le squelette d'un témoin du déluge (homo diluvii testis), mais un siècle après sa découverte, Cuvier y reconnut une espèce de salamandre, genre voisin de celui des crapauds. Aux carrières de Brugelettes dans le Hainaut, un squelette d'homme pétrifié, comme l'appelaient les ouvriers, servait de cible pour l'exercice du tir au fusil et la tête était le but vers lequel on dirigeait les balles; M.r Drapiez le classa parmi les congenères de ces tortues franches qui vivent aux Antilles. On se rappelle encore l'histoire de l'homme fossile assis sur un cheval pétrifié, découvert en 1823 dans la forêt de Fontainebleau par quelques écoliers et que M.r Huot reconnut être un bloc de grès en état de disgrégation et que le hasard avait grossièrement moulé d'après le simulacre d'un homme. En 1820 on disait avoir trouvé à Marseille un autre homme pétrifié ; c'était une 


\section{( 3 )}

empreinte de tuyaux marins. Les journaux du mois d'Août 1832 ont annoncé qu'un voyageur avait découvert aux environs de Rome, dans une forêt fossile souterraine, un chasseur et du gibier pétrifiés; de telles assertions n'ont plus besoin d'être réfutées. Dans les sablonnières du Brabant, les ouvriers prennent ces grands cylindres dont les parois sont pétries de tuyaux marins contournés, pour des ventres d'hommes dont les intestins sont devenus pierres (1).

Il n'y a pas long-tems encore que la question de l'existence des hommes fossiles se bornait à démontrer qu'avant l'époque du dernier grand cataclysme, l'espèce humaine n'avait pas encore apparu sur le globe terrestre. Dans l'état où se trouvait alors la géologie, Cuvier, dans son immortel discours sur les révolutions du globe, affirmait seulement qu'on n'avait pas encore trouvé d'ossemens d'homme parmi ceux des anciennes races éteintes, comme celles des Palæotherium, des éléphants, des rhinocéros; et ce grand naturaliste concluait dans la rigueur du raisonnement qui lui était propre, que si l'espèce humaine n'avait pas existé dans les lieux où les restes de ces races éteintes avaient été enfouis à l'époque des révolutions, l'homme pouvait bien avoir vécu avant elle dans d'autres localités. Pour lui, l'établissement du genre humain sur la majeure partie de l'Europe, de l'Asie et de l'Amérique pourrait avoir été postérieur au tems du dernier cataclysme ou du phénomène qui avait mis à sec ces mêmes continens. Cette explication était d'autant plus ingénieuse qu'au tems où elle fut proposée, et encore jusqu'après 1826 , on admettait en géologie la brusque interruption entre

(1) Voyez Burtin, Oryctographie de Bruxelles. 


\section{(4)}

l'époque anti-diluvienne et celle de nos jours ou l'époque jovienne comme on l'appelle, et l'on doit remarquer que cette opinion ne froissait en rien les croyances bibliques. Pour Cuvier, il n'y avait nulle raison pour ne pas attribuer la rédaction de la Genèse à Moïse luimême, comme il le dit, et son système sur la première origine de l'homme lui permettait de concilier la rélation du déluge mosaïque et l'idée que l'homme n'avait habité, avant cette catastrophe, que des contrées peu étendues d'où il aurait peuplé ensuite toute la terre après le repos des élémens.

Avec cette opinion s'accordaient d'ailleurs toutes les découvertes faites jusqu'alors d'ossemens d'hommes et de débris de leur première industrie, gisants dans les terrains alluviens ou d'une origine qui leur est contemporaine. C'est, en effet, dans cette classe que se rangent les squelettes humains, prétenduement fossiles, de la Guadeloupe, dépôsés dans un tuf calcaire qui se forme encore tous les jours, et ceux de la caverne des morts près de Durfort, dans le département du Gard, percée dans un calcaire secondaire et laissant incruster d'un suc de chaux les nombreux ossemens qu'elle renferme (1). Les ossemens humains trouvés à Koestriz, en Saxe, étaient dépôsés dans une argile qui remplissait les fentes d'un calcaire secondaire et se trouvaient mêlés à d'autres os d'animaux tels que de ruminans, de souris et de quelques oiseaux. Dans les crevasses du gypse de l'Elster, un phénomène analogue se répète, de même qu'à Scaham-Dene, en Angleterre, où les restes humains mêlés à ceux de cerfs, d'autres

(1) D'Honbres-Firmac, Bibl. univ. de Genève. - Marcel de Serres, Mém. de la soc. Linn. de Paris. 1824. - Renaux, Bull. des sc. nat. 1830. fom. XXII. page 28. - Marcel de Serres, Journ. dé yéul. 1830. tom. H. p. 184. 
quadrupèdes et d'oiseaux, se trouvent sous la terre végétale et sous une couche argileuse de quinze pieds, au milieu de crevasses percées dans une roche calcaire. Toutes ces découvertes n'indiquaient que des phénomènes très-circonscrits et purement locaux; les trouvailles des instrumens de silex, quarz, jaspe, jade etc., les haches, les coins, les casse-têtes, les armures de flèches déterrées sur toute la superficie du globe, hors des terrains certainement alluviens, venaient à l'appui des inductions précédentes.

Le problême des hommes fossiles semblait résolu : décidément on ne les rencontrait jamais dans les terrains régulièrement stratifiés, dans les terres vierges de tout contact humain; et notre espèce avec celle des singes, paraissait être la dernière création de la nature, le chef-d'œuvre d'une force productive éteinte depuis notre existence et comme épuisée après ce prodigieux enfantement. Mais à mesure que la géologie s'enrichit de nouveaux et de féconds principes, la question des hommes fossiles changea de face. Quand les travaux de M.r Constant Prévost eurent démontré qu'il y avait une transition insensible entre les phénomènes géologiques contemporains et ceux des époques anciennes, et que la distinction des tems anté- et postdiluviens était relative aux lieux, aux continens, aux peuples, aux sociétés, et non au tems considéré en lui-même, il ne fallut que quelques mois pour mettre la discussion des ancêtres du genre humain sur un autre terrain. Les découvertes se succédèrent avec une rapidité étonnante et eurent d'autres résultats dans la philosophie générale de l'histoire naturelle.

Les recherches de Cuvier et de M.r De Blainville sur le dronte, ce grand oiseau qui, il n'y a guère plus de deux siècles, vivait encore à l'île de France et à l'île 
Bourbon, et dont la race s'est ainsi éteinte sur le globe au milieu des tems historiques, celles de M.r Geoffroy de S.t Hilaire sur deux espèces de crocodiles dont les momies embaumées dans les catacombes de l'Égypte attestent qu'elles aussi ont disparu de notre terre, ces recherches, dis-je, prouvèrent que des races peuverit disparaître du monde animé, de la série des êtres, sans que l'homme les ait détruites et sans que l'ordre de la nature s'intervertisse par leur perte, comme le prétendaient les philosophes du XVIII. ${ }^{m e}$ siècle. Or, les découvertes faites dans les grottes du Midi de la France, d'ossemens humains mêlés à des ossemens d'ours, d'hyènes, de cerfs, etc., que les naturalistes considéraient comme dûment fossiles, parceque ces espèces n'existent plus du tout aujourd'hui, ont fait croire à quelques savans que si les ossemens de ces hommes qui avaient vécu en même tems que ces animaux, ne devaient pas être regardés comme fossiles, il fallait admettre que les races de ces ours, de ces hyènes, de ces cerfs etc. avaient disparu, de la même manière que s'étaient éteintes depuis des époques connues celles du dronte et des crocodiles de l'Égypte. M.r Marcel de Serres fut même conduit ainsi à définir comme fossiles celles des espèces animales qui avaient vécu avant l'existence de l'homme, pétition de principe qui certes fera qu'il n'y aura jamais d'hommes fossiles. M.r Tournal et d'autres géologues admettent au contraire que ces os d'hommes, trouvés dans les cavernes du midi de la France, sont bien fossiles et contemporains des espèces éteintes avec lesquelles ils ont été découverts. M.r'Teissier qui en 1831 visita avec M. Marcel de Serres les cavernes des environs d'Anduze, dans le département du Gard, avait conclu de ses recherches que soit que les ours et les autres espèces 
perdues eussent vécu dans les grottes, soit qu'ils se fussent tenus aux environs, leurs ossemens ont toujours été introduits dans les cavités souterraines par.un véhicule aqueux, un limon gras, et que les eaux en ont vidé plus tard les couloirs par un mouvement contraire; que poir les ossemens humains et les ustensiles qui les accompagnaient, il élait probable que ce furent des Romains et des Gaulois qui n'avaient pu vivre dans ces grottes ni en même tems que les ours, ni les uns avec les autres; que leur ensevelissement a été produit par la même cause qui avait introduit les restes d'ours dans les cavités souterraines. Sans assurer que les faits se soient passés ainsi, M.r Teissier croit que d'abord les ours ont habité les cavernes et les ont remplies de leurs débris, puis, que les indigènes se sont emparés de ces demeures, $y$ ont laissé leurs dépouilles, que plus tard les Romains eux-mêmes s'y sont établis pour s'y ensevelir à leur tour, et qu'enfin après ces successions, une inondation a rassemblé tous ces os, les a mêlés au limon et les a enterrés dans les cavités et les couloirs des grottes (1). Cette ingénieuse explication, très-différente de celle de M.x Buckland qui admettait que les hyènes et les autres carnassiers des cavernes avaient trainé dans leurs demeures les cadavres de leurs victimes, a été singulièrement appuyée par un mémoire géologico-historique de M.r Desnoyers (2). Ce savant croit aussi que c'est postérieurement aux cataclysmes que les ossemens humains furent dépôsés dans ces retraites souterraines et qu'ils

(1) Teissier, Bull. de la soc. géolog. de France. tom. II. page 62-63. 1831-1832.

(2) Le Temps. Fuuilleton du 9 Février 1832. - Bulletin de la soc. géuloy. de France. tom. II. page 126. 1831-1832. 
ne sont nullement contemporains des grandes espèces de mammifères que l'on trouve réunies avec eux. Il s'appuie surtout du témoignage de Florus, historien romain du II. ${ }^{\text {me }}$ siècle, qui rapporte que César avait ordonné d'enfermer les Aquitains dans les cavernes où ils demeuraient; leurs restes s'y seront confondus, dit M.r Desnoyers, avec ceux des animaux de race éteinte, et dans quelques endroits, les stalagmites auront réuni et cimenté ces ossemens de diverses espèces. Peıdant les invasions des Goths, des Sarrasins, des Francs, des Angles, des Normands, les malheureux habitans des contrées attaquées se retiraient de même dans les cavernes et les antres. Comme le fait observer M.r Desnoyers, Eginhard, le secrétaire et l'ami de Charlemagne, parle aussi de la manière dont se défendirent. contre Pepin, les Aquitains et les Wascons retirés dans leurs grottes. Encore aujourd'hui, continue le géologue français dont nous analysons ici l'intéressant mémoire, quinze à vingt mille familles du département de Loire et Cher, d'Indre et Loire, de Maine et Loire, habitent les cavernes percées dans la craie tufeau. Nous pouvont ajouter en passant que dans la vaste forêt d'Herheumont, située au milieú de la partie la plus montagneuse et la plus ancienne du schiste de transition du duché de Luxembourg, nous avons vu également des familles habiter ou des antres creusés des mains de la nature ou des exploitations d'ardoises abandonnées depuis long-tems.

En 1830, le savant M.r Ami Boué dans son compte rendu des progrès de la géologie, avait déjà embrassé la presque totalité de ces recherches et s'était demandé si la race humaine avait précédé la formation des terrains d'alluvion ou si elle était contemporaine de l'époque tertiaire el secondaire? L'existence de l'hornme 


\section{(9)}

aux époques antéalluviales ne saurait soulever de discussion; mais il faut examiner des faits plas nombreux et plus difficiles quand il s'agit de savoir si cette existence a été contemporaine du transport des alluvions. Et d'abord, on doit ajouter aux assertions de M.rs Marcel de Serres, Christol, Tournal, Farine etc., rapportées plus haut, les observationis suivantes que j'extrais du résumé de M.r Boué (1). Donati et M.r Germar attestent la présence des os humains, et M.r Keferstein celle d'un morceau de verre dans les brèches osseuses de la Dalmatie. Le comte Razoumovski a trouvé un crâne humain et d'autres os au milieu d'un détritus calcaire et de terre noire dans le calcaire alpin à Baden en Basse-Autriche. Le comte Breuner a rencontré des crânes semblables dans le sol marno-alluvial coquillier de Krems dans le même pays. Les ossemens de Koestritz, dont nous avons déjà parlé, rentrent dans la même catégorie. Enfin, M.r Ami Boué lui-même a observé en 1823 , derrière $L a h r$, dans le pays de Bade, des ossemens humains contenus dans le dépôt alluvial de la vallée du Rhin et accompagnés de coquilles appartenant à des espèces vivantes; il faut remarquer pourtant que ce dépôt renferme aussi des restes de quadrupèdes perdus. Le digne secrétaire des relations étrangères de la société géologique de France, M.x Boué, croit que ces divers faits doivent être bien étudiés pour nier ou approuver l'existence de l'homme diluvien, et cette circonspection chez un savant dont l'érudition est aussi vaste qu'éclairée, doit engager les naturalistes à fournir à la science tous les renseignemens qui pourraient éclaircir cet intéressant problême.

(1) Boué. Le Globe. 2 Décembre 1829. - Ann. des sc. nat. Décembre 1829. - New Edimb. phil. journ. Janvier 1830. - Bull. des sc. nat. tom. XX. page 195. - Bull. de la soc. géol. tom. I. page 105, 1830. 
L'histoire des événémens scientifiques que nous venons de passer en revue nous porte donc à nous attacher à l'étude des faits suivans :

1. Dans quels terrains rencontre-t-on les ossemens humains?

2. Ces ossemens y sont-ils accompagnés d'autres. restes de races éteintes?

Et ces faits étant connus, reste la partie problématique:

3.• Ces ussemens sont-ils contemporains de ces races perdues?

Nous avons vu comment les écrivains se sont divisés sur ces questions dans l'étude des terrains ossifères des cavernes. Dans nutre pays on pourra appliquer ces recherches à la connaissance des faits découverts par M.r le docteur Schmerling, de Liège, dans les deux cavernes d'Engis et d'Engihoul (province de Liège), où gisaient des ossemens humains à côté de débris d'ours, d'hyènes et de rhinocéros (1). Dans les Flandres il se présenté une série de faits analogues, quoique très-différens pour le gisement; je veux parler des ossemens humains des tourbières, et c'est à eux seulement que je veux appliquer, pour le moment, les questions précédentes.

Si les tourbières, abondamment répandues dans nos fertiles contrées, ne renfermaient que des ossemens d'animaux contemporains et vivant encore aujourd'hui dans notre pays, la trouvaille de vestiges humains parmi ces restes n'aurait qu'un médiocre intérêt et n'occuperait les savans que pour décider si la conformation de ces anciens os et du crâne, par exemple, est encore la même que celle des habitans actuels; si

(1) Bull. de l'acadèmie royale des seiences el belles-lettres de Bruxelles. page 7.1832 


\section{(11)}

ces os ne sont pas ceux de quelque soldat romain combattant sous César ou de quelque indigène vaincu, etc. Mais, comme nous allons le voir bientôt, les recherches sur les restes osseux de l'homme trouvés dans les tourbières peuvent mener à des considérations importantes pour la géologie et l'histoire des animaux.

En effet, les tourbières contiennent dans notre pays, comme dans beaucoup d'autres, des ossemens d'animaux dont les races sont éteintes ou dont le nombre d'individus actuellement existans est tellement restreint qu'on peut croire à leur prochaine disparition de la série des êtres. D'autres espèces, jadis communes chez nous, et dont nous retrouvons également les vestiges dans les amas de tourbes, sont confinées dans quelques régions éloignées de l'ancien et même du nouveau continent. L'aurochs, ce boeuf aussi grand qu'un rhinocéros, portant du poil et de la laine, ayant une longue barbe au cou, un front bombé et des mamelles disposées en carré, l'aurochs vivait jadis, et quand se formaient les tourbières des rives de la Dendre, aux environs de Ninove; aujourd'hui il est confiné dans les forêts de la Lithuanie, aux monts Caucase et Krapachs, et dẹvient excessivement rare. Des espèces de cerfs ont même entièrement disparu du globe et ont laissé leurs vestiges dans les tourbières des mêmes localités. Le castor des tourbes que Cuvier a démontré être différent de celui d'Amérique et que d'autres naturalistes confondent pourtant encore avec lui, habitait les bords de nos petites rivières des Flandres et s'ensevelissait dans nos tourbes pour nous manifester un jour son antique présence dans notre pays. A côté de ces animaux on trouve les restes de loups, de chiens, de loutres, de chèvres dont les races vivent encore aujourd'hui; et c'est au mílieu de ces ossemens d'espèces, d’âge et de 


\section{(12)}

contemporanéité si différens que gisent des os d'hommes dont il importe de connaître l'histoire.

Il est donc certain que parmi les terrains où se rencontrent les ossemens humains, mêlés à des restes d'animaux, les uns de race éteinte, les autres de race vivante, il faut noter les tourbières; or, il convient d'examiner maintenant le gisement précis de ce terrain tourbeux.

Les géólogues de l'école française et à leur tête M.r Alexandre Brongniart, ont classé les tourbes parmi le premier groupe des terrains alluviens phytogènes et les ont distinguées en tourbes herbacées et en tourbes ligneuses. Les premières, presque entièrement formées de végétaux herhacés, renferment des débris d'arts humains, des restes d'animaux, les uns d'espèces vivantes, les autres d'espèces éteintes, et sont situées dans le fond de larges vallées ou dans les vallons étroits et assez élevés; le limon argileux ou sableux les accom. pagne et quelquefois les recouvre. Les tourbes ligneuses ne sont composées que de débris d'arbres ou de leurs troncs entiers; elles existent enfouies dans le sable an bord de la mer (1). Le gisement de ces deux sortes de tourbières est lẹ même et ne fait rien préjuger de leur âge relatif; cette distinction n'a rapport qu'à la nature de leurs élémens les uns ligneux, les autres herbacés.

D'autres géologues ont admis une distinction dans les tourbes qui se rapproche davantage de la manière de voir des savans belges. Reconnaissant que c'est à l'accumulation de certaines conferves, du sphagnum palustre, des prêles, des carex, etc., que la tourbe

(1) Al. Bronyniart, Tabl. des terr. qui comp. l'écorce du globe. pages 35 - 36. 1820. 


\section{(13)}

doit son origine, ils veulent que l'une espèce se forme encore tous les jours autour des petites buttes qui existent dans les vallées, ou descend même le long des vallées (1), tandis que l'autre espèce est beaucoup plus ancienne, d'une date antérieure même à celle où l'homme a pu établir son séjour dans les pays qui,la recèlent. Cette seconde espèce de tourbe se serait déposée dans de grands lacs ou marais que l'Océan, en se retirant dans son lit actuel, aurait laissés sur les contrées riveraines; ces lacs auraient été d'abord salés, puis saumâtres, puis enfin auraient présenté seulement de l'eau douce par suite de l'afflux des rivières venues de l'intérieur, et des eaux pluviales. C'est seulement dans ces sortes de tourbières que l'on trouverait des fossiles d'espèce éteinte et quelquefois des restes d'animaux qui ont établi leurs demeures actuelles dans des contrées fort éloignées.

Cette division correspond à peu près à celle que l'on établit le plus souvent dans les provinces belges où l'on reconnait deux espèces de tourbières, les élevées (de hooge veenen) et les basses (de lage veenen); les premières sont celles qui existent au sommet des plateaux de montagnes parfois élevées jusqu'à deux et trois mille pieds au dessus du niveau actuel de l'océan, et dans les vallons de ces contrées montagneuses; les secondes sont celles des grandes plaines basses qui bordent la mer. Les tourbières des Ardennes appartiennent à la première de ces divisions, les tourbières des Flandres à la seconde, mais il faut remarquer qu'attribuer ces tourbes basses aux dépôts qui se seraient formés dans des eaux salées, est une opinion qui ne peut être appuyée d'aucun fait dans les pro-

(1) Cuvier, Disc. sur les révol. du glube. page 80. 


\section{(14)}

vinces dont nous parlons ici. Les tourbières des Flandres, quoique se trouvant, par l'embouchure de l'Escaut, sur les rives duquel elles existent si communément, dans la condition des tourbières de l'Amérique du Nord, n'ont été formées que sous les eaux douces. Il faut noter ensuite que c'est principalement dans ces tourbes qu'on a trouvé tous les ossemens d'animaux et - les restes fossiles de l'espèce humaine, beaucoup plus rares dans les tourbières ardennaises.

Il convient surtout d'examiner le gisement exact des tourbières basses.

A ne consulter que les documens généraux que nous possédons sur la géologie de la Belgique, à ne s'en rapporter même qu'aux cartes publiées jusqu’à nos jours (1), on pourrait croire qu'à partir des terrains intermédiaires et crétacés qui s'étendent du S.O. au N.E. à travers le Hainaut, le Brabant Méridional et la province de Limbourg, toutes les provinces littorales des Flandres Occidentale et Orientale, d'Anvers et du Brabant Septentrional ont la même constitution géologique et n'ont pour terrain de surface que le calcaire rapporté au calcaire grossier par les géologues qui ont écrit sur ces contrées (2). Mais il faut noter que dans les Flandres et dans la province d'Anvers, s'étend vers

(1) Je ne parle ici que de trois cartes un peu générales, les seules que je connaisse sur la Belgique; ce sont celle de M.r D'Omalius d'Halloy, celle de M.rs Von Oeynhausen et Von Dechen et enfin la carte publiée cette année par M.r Jobard.

(2) C'est principalement d'après mes travaux antérieurs que M.r Boué et d'autres géologues modernes doutent beaucoup s'il faut rapporter le calcaire du Brabant au calcaire grossier parisien. Toutes les recherches que j'ai faites sur les animaux vertebrés fossiles de ce calcaire prouvent qu'effectivement le terrain brabauçon est moins ancien qu'on ne l'a cru jusfu'ici. 
le nord un terrain sablonneux beaucoup plus moderne, composé de plusieurs assises dont la plus ancienne ou l'inférieure est un sable vert, sur lequel repose tantôt un limon noir et tout pétri de coquilles d'eau douce appartenant à des espèces encore vivantes aujourd'hui, tantôt du sable blanc ou jaune qui constitue le sol des grandes bruyères de la province d'Anvers et des terres légères du pays de Waes, dans la Flandre-Orientale, sur lesquelles, je crois, d'après quelques trouvailles que j’ai faites aux environs de Malines et de Gand, les blocs erratiques se sont encore répandus. Il est évident que ce terrain sablonneux est très moderne et d'une origine alluviale; il est certain encore que les eaux de la mer n'ont pas contribué à le former et qu'il s'est déposé seulement dans l'eau douce; le creusement du canal de Gand à Terneuze et les coupes que j'ai vues près de Berchem, de Muysen et à Hanswyk (aux environs d'Anvers et de Malines) mettent cette vérité dans tout son jour. Ce terrain vient jusqu'aux murs de Gand, s'étend au nord et c'est sur son étendue que coulent le Moervaert, la Zuyd-Leede, la Lieve etc. (1), petites rivières qui sous le rapport du gisement des animaux fossiles sont très-intéressantes. Au midi de la ligne des sables que nous venons de tracer s'étend le calcaire dit grossier recouvert presque partout d'une puissante couche de l'argile diluvienne et constituant les terres fortes des agriculteurs. Il y a donc, comme terrains de surface, deux formations bien distinctes dans la contrée que nous examinons ici : l'une argileuse, ancienne, l'autre sablonneuse, récente.

(1) Voyez l'excellente carte topographique des rives de l'Escaut occidental publièe en $\mathbf{1 8 3 2}$ chez M.r Van der Maelen, à Bruxelles, et qu'on doit aux travaux des M.rs Gheldolf, frères. 
Or, il existe sur chacune de ces formations, des tourbières qui peuvent avoir une origine commune, mais dont le gisement sur le terrain sablonneux lacustre détermine singulièrement le peu d'ancienneté. Il n'y a point de différence, en effet, dans la composition des tourbes, soit qu'on les exploite de dessus l'argile alluvienne, le calcaire dit grossier, ou le sable blanc superposé au sable vert dont nous avons parlé; mais on doit remarquer que c'est principalement dans les tourbières gisant sur ce dernier terrain, que l'on a trouvé des ossemens humains, de castor, de loups, de chiens, de loutres, de chèvres etc.; l'aurochs n'a été exhumé jusqu'ici que des tourbières reposant sur le calcaire rapporté au calcaire grossier. C'est un fait qu'attestent tous les jours les découvertes que l'on fait dans la Flandre.

Il doit résulter de là cette conclusion que, si l'espèce de castor dont on trouve les ossemens dans les tourbières des bords des petites rivières que nous avons citées plus haut, est réellement comme l'a prouvé Cuvier, une espèce distincte de celle de l'Amérique, et n'existant plus actuellement, cette espèce n'a pu disparaître que du tems où vivaient déjà les hommes qui ont laissé leurs ossemens dans ces mêmes tourbières; et dès lors cette espèce de castor serait un de ces animaux qui comme le dronte, les crocodiles dont parle M.r Geoffroy de S.t Hilaire, et peut-être les ours, et les hyènes des cavernes, comme le disent d'autres savans, ont disparu de la série des êtres vivans depuis l'existence de l'espèce humaine; conclusion qui, du moins à ma connaissance, parait neuve pour le castor (1).

(1) Pour plus de certitude à l'égard du fait que j'avance ici, je publierai incessamment les dessins et les observations st.r les castors des Flandres. 


\section{( 17 )}

Il n'en est pas moins prouvé qu'à l'égard de la seconde question que nous nous sommes posée plus haut, on doit répondre que dans les tourbières des Flandres, conme dans quelques grottes du midi de la France, les restes de l'espèce humaine $\mathrm{y}$ sont accompagnés d'ossemens d'animaux de race perdue.

Les ossemens humains qu'on rencontre ainsi dans les tourbières avec ceux de quelques animaux de race éteinte et d'autres qui vivent encore aujourd'hui, y ont été nécessairement déposés après le tems où se sont formés les terrains sablonneux verts et les terrains lacustres à coquilles d'eau douce dont nous avons parlé.

Reste la troisième question: les ossemens humains des tourbières sont ils bien contemporains des races perdues dont on trouve les vestiges avec eux? ou sontils déposés postérieurement à l'inhumation de ces mêmes restes? J'ai déjà publié dans le Bulletin de la Société Géologique de France (tom. II. page 26. 1832) que les ossemens de castor ne reposent pas toujours précisement dans la tourbe, mais sur le sable qui sert de lit aux dépôts tourbeux, et qu'ainsi il se pourrait que réellement ces os fussent plus récents que la tourbe elle-même, par laquelle ils auraient passé, et qui les aurait pénétrés de la substance colorante brune. Ainsi, la seule supposition qu'on pourrait faire pour rajeunir les ossemens des hommes gisant dans les tourbières s'appliquerait également à ceux du castor, de sorte que si rien n'empêche de croire à la simultanéité de l'existence de ces deux sortes d'êtres, rien ne la prouve non plus d'une manière positive. La question de la contemporanéité des hommes et des animaux de race perdue dont les restent gisent dans les tourbières, n'est pas rigoureusement décidée, et si la même question a fait naître pour les ossemens des cavernes 
beaucoup de discussions parmi les géologues, celle-ci en pourra soulever d'autres.

Il me reste à décrire les ossemens humains trouvés dans nos tourbières et qui sont déposés au cabinet de l'Université de Gand. Ce sont deux frontaux qui ont été déterrés à Denderleeuw dans la Flandre-Orientale. Ils sont parfaitement semblables à ceux qui proviennent des tourbières situées plus au nord. Je les ai figurés (voyez la planche) et réduits à la moitié de leur grandeur.

On remarquera d'abord leur petitesse; voici leur dimensions comparées à celles du frontal ordinaire d'un Flamand :

Longueur absolue du 1.er $\stackrel{m}{0,101}$. du 2.de ${ }_{0,105}^{m}$. d'une tête ordịnaire 0,120 . Hauteur absolue . . 0,069 . . 0,063 . . . . . . 0,083 .

Largeur absolíe . . $0,106 . \quad$. 0,103. . . . . . 0,125. Lar.del'échancrurenasale 0,016. . . 0,022. . . . . . 0,024 .

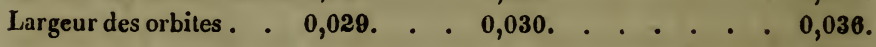

Ce n'est pas que dans les Flandres on ne rencontre beaucoup de têtes dont les frontaux ne soient aussi petits que ceux des troubières, mais le fait à remarquer est qu'aucun de ces deux frontaux ne présente les dimensions les plus ordinaires. Il faut noter encore qu'étant assez bombés ils paraissent a voir moins d'étendue. La suture médiane est soudée chez l'un et chez l'autre, de sorte que ce sont au moins des os d'adultes. Sur l'un, la ligne médiane est légèrement proéminente, sur l'autre elle ne l'est pas du tout. Le premier a la bosse nasale très-peu saillante, celle du second est à peine sensible. Les bosses frontales de celui-ci sont larges écartées, plates, ce qui donne au front un développement latéral qu'on n'observe pas dans celui-là dont les bosses frontales sont rapprochées, élevées, ce qui donne all front une forme pyramidale. Aux ar- 
cades sourcilières, il n'y a rien de remarquable, pas plus qu'à la face cérébrale. Ces os ont peu d'épaisseur. En appliquant le goniométre sur la droite qui réunit les bosses nasale et frontale, et la branche mobile sur la partie supérieure de l'os, pour avoir l'angle d'inclinaison de ces os, on trouve pour l'un $1080^{\circ}$ et pour l'autre $97 .^{\circ}$, mais on ne peut pas juger par cette mesure de ce qu'était l'angle facial sur les têtes aux quelles ils ont appartenu. Il suffit cependant d'examiner la planche ci-jointe pour s'apercevoir que cet angle ne pouvait pas être très aigu et qu'il était le même que celui des têtes des Flamands actuels.

Ainsi, toute la structure de ces têtes indique dans ces hommes de la tourbe autant de moyens d'intelligence que dans ceux des habitans actuels de nos contrées, et si ces têtes sont contemporaines de cette race de castor qui a disparu du globe, on peut croire que depuis l'époque déjà reculée de leur enfouissement dans le sol, l'organisation de l'espèce humaine dans les Flandres n'a pas changé. Les paroles du poëte

\section{Grandiaque effosis mirabitur ossa sepulcris}

ne peuvent pas non plus trouver ici leur application.

Il aurait été convenable peut-être de faire précéder ces remarques des observations qu'on a faites sur les crânes d'hommes trouvés dans d'autres localités, pour en connaître précisement la race.

Le comte Razoumovski qui a trouvé, comme nous l'avons dit plus haut, à Baden en Basse-Autriche, et dans les cavités du calcaire alpin, des ossemens humains mêlés à ceux de quadrupédes d'espèce éteinte, tels que de rhinocéros, d'ours etc., avait déjà remarqué que la forme de ces têtes humaines était différente de celle des habitans actuels du pays. Le comte Breuner, selon les écrits de M.r Ami Boué, a 


\section{(20)}

observé de même que les crânes trouvés dans le sol marno-alluvial coquillier de Krems, en Basse-Autriche, avaient une structure très bizarre; le front était très applati comme chez les races sauvages, et les crânes étaient comprimés et fort longs. M.r Edwards, l'ainé, va même jusqu'à dire que ce n'est que parmi les Caraïbes et les indigènes du Chili qu'on retrouverait de ces sortes de crânes, d'ailleurrs assez rapprochés de ceux des singes. En 1831 , M.r Teissier a découvert dans la caverne de Miallet près d'Anduce (département du Gard), creusée dans une roche dolomitique, des ossemens d'ours, d'hyènes, de ruminans, d'oiseaux et entremêlés d'os humains; près d'un squelette de ce genre se trouvaient une lampe, une figurine en terre cuite et des bracelets de cuivre; plus loin étaient des poteries, de outils de silex et des os travaillés. Les crânes étaient déprimés de haut en bas et allongés d'avant en arrière, comme dans certaines races nègres. M.r Teissier remarqua néanmoins que ces têtes étaient de la race caucasique et que la dépression du crâne indiquait seulement l'usage de porter des fardeaux sur la tête.

Si l'on laisse de côté la discussion de la cause problématique qui a produit l'applatissement de ces crânes, et si l'on se rapporte aux observations faites en BasseAutriche, il reste constant que les têtes trouvées dans des couches terrestres, assez anciennes par rapport aux tems historiques, n'ont point la même forme que celles des peuple actuels de l'Europe. Les faits ne sont pas assez nombreux pour avoir à ce sujet une opinion fixe, mais il importe beaucoup d'altirer l'attention des savans sur ce point, parce qu'il se lie à une foule de questions archéologiques et au grand systême des émigrations des peuples. C'est pour ce motif que 
nous ferons observer ici qu'il suffit d'une simple inspection pour se convaincre que les frontaux des tourbières, dont nous parlons, appartiennent indubitablement à la race caucasique, et que de plus ils ne sont pas différens pour leur configuration de ceux des Flamands actuels.

Il n'y a pas de raison pour ne pas croire que ces crânes ne soient contemporains de ceux de quelques races perdues d'animaux de tourbières, et puisqu'ils montrent avec ceux des hommes d'aujourd'hui une idendité, je dirai parfaite, ils peuvent servir à démontrer la fixité dans l'organisation humaine et l'invariabilité avec laquelle la nature conserve les espèces, principe de philosophie zoologique sur lequel les plus grands esprits n'ont pas toujours été d'accord et sur lequel ils discutent encore journellement.

Les observations qui précédent pourront, je crois, mener à des faits dont la géologie peut tirer quelques inductions intéressantes. Les conclusions de ce mémoire sont les suivantes:

1. S'il est prouvé que l'existence de l'homme sur la terre est postérieure à l'époque des terrains tertiaires, il faut reconnaître que les opinions sont partagées sur la question de savoir si l'espèce humaine a vécu ou non avant l'époque du dernier déluge; ensuite les uns disent que l'homme a pu exister avant cette époque, mais que ce n'est qu'après elle qu'il s'est établi sur les continens actuels; les autres qu'il a séjourné sur ces continens a vant et pendant la catastrophe diluvienne.

2. S'il est de fait que parmi les ossemens de grands animaux dont l'espèce est aujourd'hui éteinte et qui ont été enfouis dans les cavernes des montagnes, gisent également des ossemens humains, les géologues doutent s'il faut en conclure que l'existence de l'homme et 


\section{( 22 )}

celle de ces animaux perdus doivent être considérées comme contemporaines; s'il faut admettre par conséquent que depuis la présence de l'espèce humaine sur la terre, des races entières d'animaux aient pu disparaître et s'éteindre entièrement, comme le fait parait d'ailleurs se démontrer pour d'autres genres d'animaux qui se sont perdus depuis les tems historiques.

$3 .^{\circ}$ Si le gisement des ossemens humains mêlés à ceux d'hyènes, d'ours, de cerfs, de rhinocéros n'a été reconnu que dans les cavernes du midi de la France, dans celles de la Belgique, dans le détritus et la terre noire du calcaire Alpin de la Basse-Autriche, si les ossemens humains se sont rencontrés de même dans les brèches osseuses de la Dalmatie, dans le sol marnoalluvial de Krems et dans le grand dépôt alluvial du Rhin, il faut faire entrer, dans la même série de faits, le gisement des ossemens humains dans les tourbières, puisque les dépôts tourbeux contiennent de même des races animales qui ont disparu du globe. Il faut donc se demander si des espèces de cerfs, de castors etc. doivent être comprises dans la série des animaux qui ont eu leur race éteinte depuis que l'homme existe sur la terre.

4. L'histoire des dépôts tourbeux, considerée sous ce point de vue, réçoit une lumière nouvelle. En classant les tourbières sous le rapport de leur horizon géographique en tourbières basses et élevées, on doit reconnaître que si les premières sont effectivement plus anciennes que les autres, elles renferment, en plus grande quantité, des ossemens humains et ceux d'animaux perdus et émigrés hors du pays. On remarquera encore que ces tourbières peuvent reposer sur des terrains très différents, mais que leur superposition au sable limoneux d'eau douce renfermant des coquilles 
dont les espèces vivent encore aujourd'hui, prouve et leur peu d'ancienneté et l'impossibilité qu'elles se soient formées dans de grands lacs d'eau salée; dernière assertion de quelques auteurs, assertion dont la vérité est entièrement controuvée en Flandre par la composition même de la tourbe.

5. $\mathrm{Si}$ des ossemens humains trouvés dans quelques contrées d'Europe mêlés à ceux d'animaux éteints ont montré dans les crânes une structure qui indiquait tantôt un rapprochement avec les races nègres, les Caraïbes ou les habitants du Chili, tantôt une compression de la tête, suite de l'usage prolongé de porter des fardeaux sur cette partie du corps, on doit remarquer que les ossemens humains trouvés dans les tourbières des Flandres, et également mêlés à des os d'animaux perdus, n'ont point présenté de faits analogues. Ces os appartiennent à l'espèce humaine de la race caucasique et ne sont pas sensiblement différens de ceux des habitans actuels des Flandres.

6.0 Si l'on conclut de ces faits que par rapport à nos tems historiques, l'époque de la formation des tourbières basses et de l'enfouissement dans leurs couches de castors, de l'aurochs, de cerfs, de loups, de chiens, de loutres et de quelques ruminans est déjà très reculée, fait que semble démontrer d'ailleurs la disparition de l'espèce de castor depuis l'existence de l'homme, on pourra en conclure avec quelque certitude qu'à partir de ce tems éloigné jusqu'à nos jours l'organisation de notre espèce n'a point changé dans nos climats.

Les savans qui ont embrassé l'étude des grandes questions de la philosophie zoologique générale verront sans doute dans ces énoncés, des faits dont les conséquences pourraient être poussées plus loin.

Juillet, 1832. 


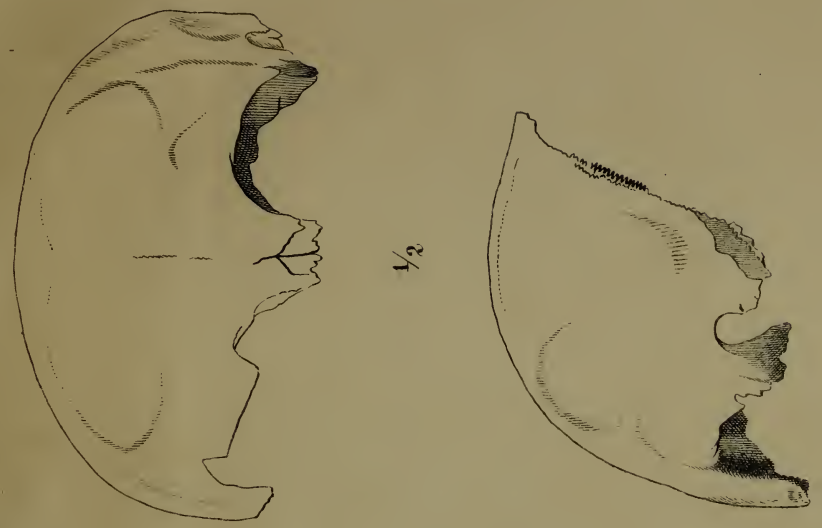

ב⿱艹
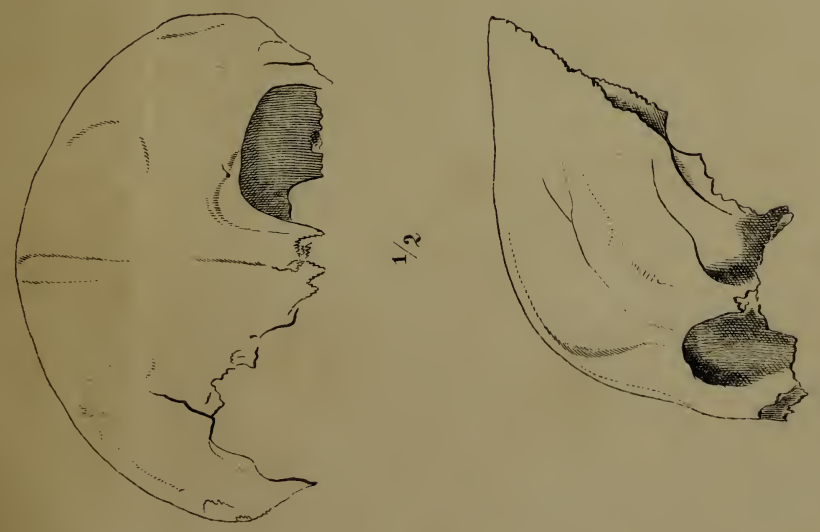





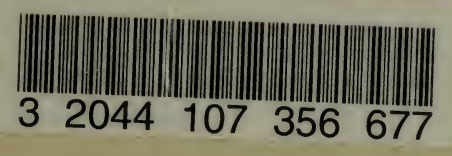


